\title{
How do Catalan Nurses See the Continuity of Care?
}

\author{
Reig Garcia G*1, Figa Vaello J², Vila Vendrell A² and Juvinyà Canals $\mathrm{D}^{3}$ \\ ${ }^{1}$ Nursing department, University of Girona, Spain \\ ${ }^{2}$ Health department, Institut d'Assistència Sanitària, Girona, Spain \\ ${ }^{3}$ Research group health and healthcare, University of Girona, Spain \\ Received: January 21, 2018; Published: February 08, 2018 \\ *Corresponding author: Reig Garcia G, Nursing department, University of Girona, Catalonia, Spain; Tel: 34630749201; Email: gloria.reig.ias.cat
}

Keywords: Nursing; perception; nursing care continuity; safety

\section{Introduction}

The Health System of the autonomous region of Catalonia (Spain) involves four main actors: the Catalan Department of Health (DoH); the Catalan Health Service (CatSalut); health providers; and citizens. The DoH is in charge of establishing health policies and maintaining levels of quality in provision of health services by setting out a health plan, distributing the healthcare budget, and accrediting health care providers. The CatSalut is the public insurer that is responsible for planning, purchasing, and assessing health services according to the population's needs. The CatSalut establishes service policies in line with the health policies defined by the DoH. The Catalan territory is divided into seven health regions. Each region is structured in health sectors which bring together the so-called Basic Health Areas formed by districts in urban areas, or one or more municipalities in rural areas. The health providers are the organisations with which the CatSalut enters into multiannual contracts the provision of care services. Contracts with health providers are revised on a yearly basis and include health objectives, activity, cost rates, invoicing systems and evaluation procedures [1].

The provision on healthcare is organised into four main levels: primary care; specialised or hospital care, social health care and mental health care. Primary care is the gatekeeper and responsible for coordinating the patient's care along the care continuum. Specialised or hospital care acts as a consultant of primary care and is responsible for more complex care [2]. The provision of healthcare is now responsibility of different providers which have different types of ownership. Increasingly, patients receive care from a variety of providers belonging to very different types of organisations and in different place. Concerns have been raised because this fragmentation of care. Policy reports and documents $[3,4]$.

Healthcare continuity is the degree of coherence and union of the experiences in the care received by the patients throughout time, which must be coherent with their health needs and personal environment [5]. The nursing care continuity is based upon the transfer of information, as well as on the coordination and consistency of care among nurses, who should adapt care according to the patient's needs and condition. A strategy is built up in order to improve the care received by patients who have been assisted in the different care levels [6-8]. The health care continuity process is multidisciplinary. However, nurses are key experts to lead the improvement of this process $[9,10]$. This article aims to improve the knowledge on nurses' perception of nursing care continuity.

\section{Methodology}

Cross-sectional descriptive study about the nurses' perception regarding the nursing care continuity on the four areas: primary care, specialized or hospital care, socio-sanitary care and mental health care. Anonymous and self-administered questionnaires were delivered. The variables were divided into two parts: sociodemographic and nurses' perception of nursing continuity of care. We asked about the perceptetion of communicate all the activities carried out during the patients hospitalisations or communicate only those activities that needs continuity of care in the other levels. Data analysis was done through a frequency descriptive expressed by average numbers and dispersion measurements: Typical Deviation and the minimum and maximum value, as well as descriptive statistics expressed by frequency and 
percentage. A bivariate analysis was done with no parametrical evidence: Kruskal-Wallis test and Mann Whitney U test and with the Pearson chi-square coefficient

\section{Results}

A representative sample of 639 nurses was asked to evaluate their perception of nursing continuity of care. The response rate was $68.5 \%(n=437), 92.4 \%$ were woman, average age was 40,5 years. To assess the perception of nurses about nursing continuity of care we asked if they thought that all activities carried out during hospitalization should be reported at the hospital discharge. 95\% of nurses considered it was important to inform all the activities carried out during hospitalization at the hospital discharge, obtaining scores significantly higher $(p<0,05)$ at socio-sanitary care.

The bivariate analysis indicated that the younger nurses (35.6 \pm 5.9 years) had a worst perception of nursing care continuity. It also showed that nurses working on the basis of permanent contracts had higher scores in perception of nursing care continuity $(p<0.05)$. Another factor was the nurses' formation. Nurses without research training considered it important to communicate nursing activities that required care continuity only (96.7\%) whereas the nurses with research training $(89 \%)(p<0.05)$ stressed the importance of communicating all the activities carried out during the patient's hospitalisation and not those requiring care continuity.

\section{Conclusion}

Nurses' perceptions about nursing care continuity are high, especially among older nurses. Lack of training in communication can be identified as a barrier to nursing perception about nursing care continuity. The fact that nurses without research training considered it important to communicate only activities that need continuity and did not consider all aspects related to the process suggests that the lack of knowledge in a so recurring research area could leave aside important aspects of the person for his or her integral care.

\section{References}

1. (2011) Cat Salut Report, Memoria del Cat Salut, Generalitat de Catalunya, Departament de Salut.

2. Aanestad M, Grisot M, Hanseth Ole (2017) Information Infrastructures within European Health care: Working with the Installed Base.

3. The Ljubljana charter on reforming health care (1996) World Health Organization, Switzerland.

4. Canadian Health Services Research Foundation (2001) Open grants competition: call for letters of intent, Canada.

5. Reid R, Haggerty J, McKendry R (2002) Defusing the confusion: concepts and measures of continuity of healthcare. Ottawa: Canadian Health Services Research Foundation.

6. Carter M, Bower P, Gask L, Greco M (2004) Improving your practice with patient surveys. National Primary Care Research \& Development Center, England.

7. Haggerty J, Reid R, Freeman GK, Starfield BH, Adair CE et al. (2003) Continuity of care: a multidisciplinary review. Br Med J 327(7425): $1219-1221$.

8. Anderson MA, Helms LB (2000) Talking about patients: communication and continuity of care. J Cardiovasc Nurs 14(3): p15-28.

9. Less L (2004) Making nurse-led discharge work to imporve patient care. Nursing times 100(37): 30-32.

10. Yilmaz M, Emirouglyu O (2005) The need assessment of MI Patients in discharge Planning and home-health care: a sample from Turkey J Adv Nurs 7(2): 1-14.
This work is licensed under Creative Commons Attribution 4.0 License

Submission Link: http://biomedres.us/submit-manuscript.php

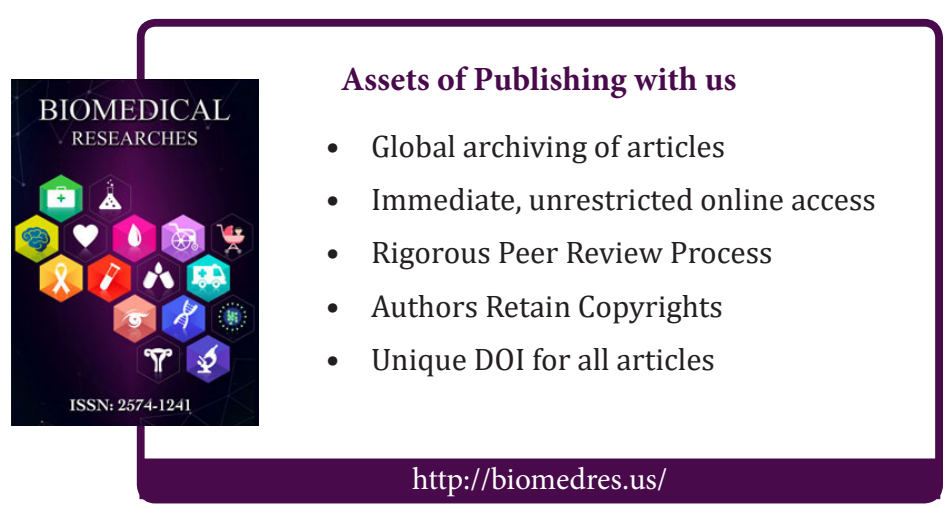

